\section{ANÁLISE HISTOMORFOMÉTRICA DA ARTÉRIA AORTA DE RATAS SUBMETIDAS À ADMINISTRAÇÃO REPETIDA DA ASSOCIAÇÃO DE ETINILESTRADIOL E GESTODENO}

\section{HISTOMORPHOMETRIC ANALYSIS OF THE AORTA OF RATS SUBMITTED TO REPEATED ADMINISTRATION OF ASSOCIATION OF ETHINYLESTRADIOL AND GESTODENE}

\author{
Geovane Ribeiro dos Santos ${ }^{1,2}$, Talita Aparecida Lazarini1,2, \\ Jessyca Cristina Ramos Ferreira ${ }^{1,2}$, Raphael Cruz Seabra Prudente ${ }^{1}$, \\ Marcelo Rodrigues da Cunha' ${ }^{1}$, Clóvis Antônio Lopes Pinto ${ }^{1,3}$
}

\section{RESUMO}

Introdução: Os anticoncepcionais orais vêm sofrendo alterações em suas concentrações de estrogênio e progestogênio, baseando-se no fato de que doses mais baixas estão associadas a menor incidência de alterações metabólicas e de efeitos adversos, como o comprometimento da morfologia e função da artéria aorta. O objetivo deste estudo é avaliar a influência dos anticoncepcionais nas propriedades morfofuncionais da artéria aorta, através do método histoquímico, demonstrando sua utilidade nas análises histopatológicas.

Métodos: Para tanto, foram utilizadas 15 ratas divididas em 3 grupos; Grupo controle (GC), cujos animais não receberam tratamento hormonal; Grupo 2 (G2), onde os animais receberam tratamento diário de $15 \mu \mathrm{g}$ de etinilestradiol $+60 \mu \mathrm{g}$ de gestodeno; e Grupo 3 (G3), que recebeu $30 \mu \mathrm{g}$ de etinilestradiol $+75 \mu \mathrm{g}$ de gestodeno. Após o tratamento, os animais foram eutanasiados e as artérias retiradas para análise histológica.

Resultados: Os valores encontrados com a técnica de coloração histológica de hematoxilina e eosina ( $\mathrm{HE}$ ), bem como na coloração específica em histoquímica com Alcian Blue, demonstram que no G3, tratados com uma dosagem superior de hormônio, $8 \%$ das ratas tem aumento relevante da espessura de suas artérias, ou seja, superior ao G2, que recebeu dosagem menor de hormônio, e GC, o qual não recebeu nenhum tipo de tratamento.

Conclusão: Este estudo demonstra que existem relações entre o espessamento das artérias, de ratas submetidas a dosagens de etinilestradiol e gestodeno, com a grande quantidade de mucopolissacarídeos depositados entre as túnicas das artérias.

Palavras-chave: Contraceptivos; fármacos; histoquímica; morfologia; vasos sanguíneos

\section{ABSTRACT}

Introduction: Pharmacological development of hormonal contraceptives has been undergoing changes in estrogen and progesterone doses, based on the fact that lower doses are associated with a lower incidence of metabolic changes and adverse effects, such as impairment of morphology and function of the aortic artery. The aim of this study is to evaluate the influence of contraceptives on the morphofunctional properties of the aortic artery, using the histochemical method, demonstrating its usefulness in histopathological analyzes.

Methods: For this purpose, 15 rats were divided into 3 groups; Control group (GC), whose animals did not receive hormonal treatment; Group 2 (G2), where the animals
Clin Biomed Res. 2018;38(2):141-150

1 Departamento de Morfologia e Patologia Básica, Faculdade de Medicina de Jundiaí (FMJ). Jundiaí, SP, Brasil.

2 Instituto de Ciências da Saúde (ICS), Universidade Paulista (UNIP). Jundiaí, SP, Brasil.

3 Serviço de Anatomia Patológica, Hospital A.C. Camargo. São Paulo, SP, Brasil.

Autor correspondente:

Clóvis Antônio Lopes Pinto patologia@fmj.br

Departamento de Morfologia e Patologia Básica, Faculdade de Medicina de Jundiaí (FMJ)

Rua Francisco Teles, 250.

13202-550, Jundiaí, SP, Brasil. 
received a daily treatment of $15 \mu \mathrm{g}$ of ethinylestradiol $+60 \mu \mathrm{g}$ of gestodene; and Group 3 (G3), whose animals received $30 \mu \mathrm{g}$ of ethinylestradiol $+75 \mu \mathrm{g}$ of gestodene. After treatment, the animals were sacrificed and the arteries removed for histologic analysis.

Results: The values found, with HE measurements and Alcian Blue histochemical staining, showed that in G3, treated with a higher hormone dosage, there are $8 \%$ of the rats with a relevant increase in the thickness of their arteries, that is, higher than that of G2, which received lower dosage of hormone and that of GC, which received no treatment.

Conclusion: This study demonstrates that there are relations between the thickening of the arteries of rats submitted to dosages of ethinylestradiol and gestodene with the great amount of mucopolysaccharides deposited between the tunics of the arteries.

Keywords: Contraceptives; drugs; histochemistry; morphology; blood vessels

Os anticoncepcionais hormonais, incluindo os combinados orais, são os métodos contraceptivos reversíveis mais eficientes disponíveis e, ao mesmo tempo, os mais utilizados ${ }^{1}$.

O desenvolvimento dos contraceptivos orais evoluiu para a diminuição das doses de estrogênio e progestagênio, baseando-se no fato de que doses mais baixas estão associadas a menor incidência de alterações metabólicas e de efeitos adversos².

Gestodeno é um agente progestógeno, e este hormônio faz parte da composição de contraceptivos orais trifásicos de dose reduzida. A preparação trifásica imita a produção variável de hormônios do ciclo menstrual da mulher, mantendo a dose total dos hormônios exógenos tão baixos quanto possível, prevenindo a gravidez de forma confiável e conduzindo a uma diminuição significativa da duração e intensidade das hemorragias de privação ${ }^{3}$.

O etinilestradiol é o principal estrogênio endógeno nos seres humanos. É um estrogênio bioativo utilizado por via oral, usado em muitas formulações de pílulas anticoncepcionais orais combinadas. É um dos medicamentos mais comumente utilizados ${ }^{3}$.

Os contraceptivos orais combinados, que possuem 2 hormônios em sua composição, o etinilestradiol e o gestodeno, agem por supressão das gonadotropinas, ou seja, pela inibição dos estímulos hormonais que levam à ovulação ${ }^{3}$.

O uso desses contraceptivos está associado ao aumento do risco de eventos tromboembólicos (formação e eliminação de coágulos nos vasos sanguíneos) e trombóticos (obstrução de algum tipo de veia ou artéria) ${ }^{4}$. Entre os eventos relatados estão infarto do miocárdio e acidentes vasculares cerebrais ("derrame"), ataque isquêmico transitório (paciente apresenta sintomas de derrame que duram menos de 24 horas: diminuição de força, dificuldade de falar, alteração de coordenação, diminuição de sensibilidade $)^{4}$. O risco para tais eventos é ainda maior em mulheres com condições predisponentes para tromboembolismo e tromboses venosas ${ }^{5}$.

A artéria aorta é a maior e principal artéria do organismo, ela recebe todo o sangue ejetado do ventrículo esquerdo do coração, distribuindo-o para todo o corpo. A aorta ramifica-se em artérias menores ao longo de seu trajeto, desde o ventrículo esquerdo até a parte inferior do abdômen, ao nível da porção superior do osso do quadril. Sua parede apresenta três elementos principais, a camada íntima (revestimento interno em contato direto com o sangue), a camada média (muscular) e a camada adventícia (camada mais externa), camadas também conhecidas como túnicas ${ }^{5,6}$.

As túnicas arteriais podem apresentar alterações, como o aumento entre a membrana elástica interna e a muscular e o aumento do tamanho das fibras musculares lisas que formam a túnica média da parede arterial, gerando hiperplasia. Com o espessamento da túnica média arterial, ocorre a expressão da alfa-actina no músculo liso, permanente ou transitoriamente ${ }^{7,8}$.

Os processos degenerativos arteriais podem ser classificados em arterioscleróticos e ateroscleróticos, sendo a aterosclerose uma variante morfológica da arteriosclerose. O termo "arteriosclerose" designa o endurecimento das artérias e é relacionado a uma gama de processos que englobam o espessamento arterial e a perda de elasticidade das artérias ${ }^{9}$. Em humanos, verificou-se que, quando o endotélio vascular perde a capacidade de manter um equilíbrio entre as túnicas, a camada íntima fica exposta e a aterosclerose evolui, provocando um espessamento da camada média. $O$ estresse é um dos principais responsáveis por esse espessamento das camadas arteriais $^{10}$.

Embora qualquer artéria possa ser afetada, os principais alvos são as artérias aorta, as artérias coronárias e cerebrais, tendo como principais 
consequências o infarto do miocárdio, a isquemia cerebral e o aneurisma aórtico ${ }^{11}$.

Quando o endotélio vascular perde a capacidade de manter um equilíbrio entre as túnicas, a camada íntima fica exposta e doenças evoluem, provocando um espessamento da camada média, desencadeando uma série de alterações fisiológicas que podem refletir em lesões vasculares, com possível aumento de pressão arterial ${ }^{11}$.

Historicamente, uma grande preocupação em torno do uso de contraceptivos orais era o aumento de eventos cardiovasculares associados ao uso da pílula. Este problema, contudo, ocorria com mais frequência décadas atrás, quando as pílulas eram compostas por elevadas doses de hormônios. As taxas da doença aumentaram significativamente com o uso em longo prazo das pílulas, aumentando de 20 a $30 \%$ por década de utilização ${ }^{5,12}$.

A probabilidade de sofrer de arteriosclerose aumenta com a idade e suas complicações podem ser fatais. Quando as placas se desprendem das paredes dos vasos sanguíneos, podem causar a formação de coágulos, bloqueando o fluxo de sangue ou migrando para outras partes do corpo ${ }^{5,6}$.

Os coágulos bloqueiam as artérias que levam sangue ao coração ou ao cérebro, podendo causar infartos ou derrames cerebrais ${ }^{5,6,12}$. Um dos métodos utilizados para avaliar essas alterações, e o estudo de anatomia patológica, com uso das colorações de hematoxilina e eosina $(\mathrm{HE})$, e as diversas colorações em histoquímica, entre elas Alcian Blue e Verhoeff ${ }^{13}$.

Os cortes histológicos são submetidos à coloração de Alcian Blue, para coloração de mucopolissacarídeos em azul. O corante utilizado de Alcian Blue é um corante básico usado não somente para a identificação de mucopolissacarídeo, mas também para marcar as cartilagens e outras estruturas corporais. Para muitos estudos são os mais utilizados como corante catiônico, tanto para microscopia ótica como eletrônica ${ }^{14-16}$.

Lesões coronarianas foram relatadas em algumas espécies, sendo um dos principais corantes para avaliação o Alcian Blue. No presente estudo, exames foram realizados evidenciando lesões arteriais caracterizadas por hiperplasia das túnicas das artérias coronárias. A análise evidenciou alterações na estrutura das túnicas, sinais de hiperplasia e obstrução luminal, e acúmulo de proteoglicanos na análise histopatológica com coloração histoquímica com Alcian Blue, o que demonstra a presença de alterações na composição da matriz extracelular ${ }^{11}$.

Verhoeff é um método utilizado para demonstração de fibras elásticas, colágeno e outras estruturas em cortes histológicos. As fibras elásticas são coradas em azul/preto e o fundo em amarelo ${ }^{13,14,16}$.
As fibras elásticas são formadas por uma proteína chamada elastina, que está presente nas artérias elásticas e normalmente têm uma coloração amarelada. A elastina se caracteriza por formar fibras mais finas que aquelas formadas pelo colágeno, fibras as quais cedem bastante à tração, mas retornam à forma original quando é cessada a força. Essa propriedade é responsável pela manutenção da pressão sanguínea nos períodos de diástole do ventrículo esquerdo, ou seja, quando o sangue não está saindo do coração ${ }^{13-16}$. As fibras elásticas não se coram bem com HE, sendo preciso recorrer a outros métodos como a Verhoeff e Fucsina Resorcina ${ }^{16}$.

O objetivo desse estudo foi avaliar a influência dos anticoncepcionais de ultrabaixa e baixa dosagem nas propriedades morfofuncionais da artéria aorta. Demonstrar a importância do método histoquímico e o quanto se torna útil para essa análise.

\section{MÉTODOS}

\section{Obtenção dos Hormônios}

Foram utilizados contraceptivos de etinilestradiol e gestodeno em forma de comprimido. O fármaco foi triturado e misturado na ração Purina em forma de pasta ${ }^{17}$. O consumo desta dieta sólida com o medicamento foi ministrado aos animais dos grupos tratados experimentalmente por 20 semanas.

\section{Planejamento Experimental}

Foram utilizadas 15 ratas (Rattusnorvegicus, Wistar), com aproximadamente 8 semanas de idade e peso médio de $150 \mathrm{~g}$. Foram mantidas durante todo o período experimental em ambiente monitorado tendo a temperatura controlada $\left(23 \pm 1{ }^{\circ} \mathrm{C}\right)$ e ciclo claro/escuro de 12/12 horas.

Este projeto de pesquisa foi aprovado pelo Comitê de Ética em Experimentação Animal (CEUA) da Faculdade de Medicina de Jundiaí (Protocolo 134/2016 CEUA/FMJ).

Os animais foram distribuídos em 3 grupos com 5 animais cada, como demonstrado a seguir:

Grupo 1 (GC): Animais controle, tratados com dieta sólida sem diluição dos hormônios;

Grupo 2 (G2): Animais tratados diariamente com 20 gramas de ração pastosa com $15 \mu \mathrm{g}$ de etinilestradiol + $60 \mu \mathrm{g}$ de gestodeno;

Grupo 3 (G3): Animais tratados diariamente com $20 \mathrm{~g}$ de ração pastosa com $30 \mu \mathrm{g}$ de etinilestradiol $+75 \mu \mathrm{g}$ de gestodeno.

Os animais foram mantidos em gaiolas individualizadas para o controle exato do consumo da ração e do medicamento nos casos dos grupos 2 e 3 . Todas as ratas receberam água ad libitum. Houve monitoramento constante dos animais durante os 
procedimentos, tomando os cuidados com o estresse e a dor. Após o tratamento com os hormônios, os animais foram eutanasiados e a artéria aorta foi retirada para as análises do tecido.

\section{Análise Histológica}

A artéria aorta de cada animal foi imersa em formol tamponado a 10\%. Em seguida, as amostras foram submetidas ao processador de tecidos, com o objetivo de desidratar e diafanizar as mesmas. Tal procedimento é realizado em um período de 12 horas, onde o material foi submetido a 01 banho de formol, 06 de álcoois, 01 de álcool/xilol, 02 de xilóis e, por fim, 02 banhos de parafinas a $65^{\circ} \mathrm{C}$.

Quando a amostra foi retirada do equipamento, a mesma foi inclusa em parafina, o que possibilitou seções de cortes do tecido com $4 \mu \mathrm{m}$ de espessura. Foram produzidas 03 lâminas por amostra, cada amostra foi submetida a um corante diferente, a bateria de coloração histológica do HE, a especifica de histoquímica com Alcian Blue, para marcação de depósitos de mucopolissacarídeo, e a com Verhoeff para marcação da fibras elástica ${ }^{8-10 .}$

\section{Análise Microscópica}

Com lâminas devidamente prontas, as mesmas foram analisadas e fotografadas no microscópio de luz com sistema de captura de imagens, onde todas as observações de análise foram realizadas com objetivas planacromáticas.

\section{Análises Estatísticas}

Os valores obtidos foram submetidos ao teste de correlação de Pearson. Esta análise obteve nível de confiança acima de 95\%, com $p<0.0001$.

\section{RESULTADOS}

Ao realizarmos as aferições nos cortes histológicos das artérias coradas em $\mathrm{HE}$, analisamos as medidas de área externa (Figura 1A), interna (Figura 1B) e diâmetro, bem como a medida de espessura linear distribuída em 08 pontos (Figura 1C).

Os valores encontrados demonstram que no G3, tratado com uma dosagem superior de hormônio, há $8 \%$ das amostras com aumento relevante da espessura de suas artérias, ou seja, superior ao G2, que recebeu dosagem menor de hormônio, e ao

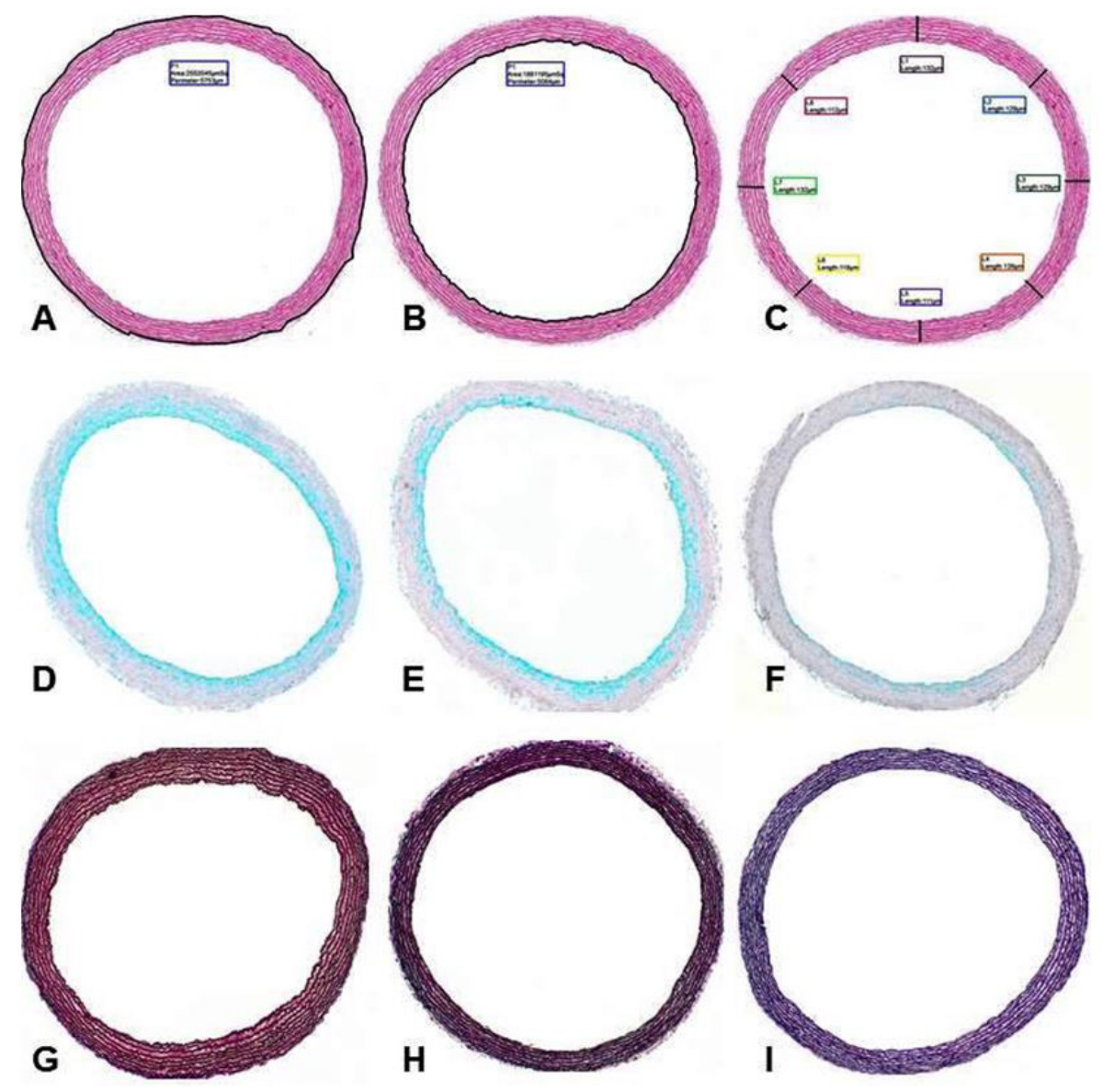

Figura 1: Fotomicrografias de cortes histológicos das artérias aorta (A, B e C) Coloração de HE; (D, E e F) Coloração de Alcian Blue; (G, H e I) Coloração de Verhoeff. Aumento de 40X. 
GC, o qual não recebeu nenhum tipo de tratamento. Já os valores do $\mathrm{G} 2$, apresentaram $4 \%$ das amostras com espessuras de artérias semelhantes ao do G3, $4 \%$ mantiveram os valores superiores ao GC e $2 \%$ apresentaram valores de espessuras das artérias semelhantes ao GC.

Nas análises histoquímicas de Verhoeff, para estudos das fibras elásticas em microscopia óptica (Figuras 1G, H e I), não evidenciamos alterações significantes entre os grupos estudados. Entretanto, para uma análise mais objetiva, recomendamos métodos de pesquisa com captura de imagem computadorizada.
Com relação à análise histoquímica de Alcian Blue, para marcar depósito de mucopolissacarídeo (Figuras 1D, E e F), as análises foram feitas com imagens submetidas à edição digital que permitiu contraste de cores, realçando os tons em azul da coloração, as quais, foram analisadas por três avaliadores distintos.

As imagens da coloração do Alcian Blue foram analisadas e organizadas da maior expressão para a menor e identificadas em ordem alfabética da letra A à letra $\mathrm{O}$ (Figura 2), para que os avaliadores não soubessem o grupo ao qual pertencia cada amostra analisada.

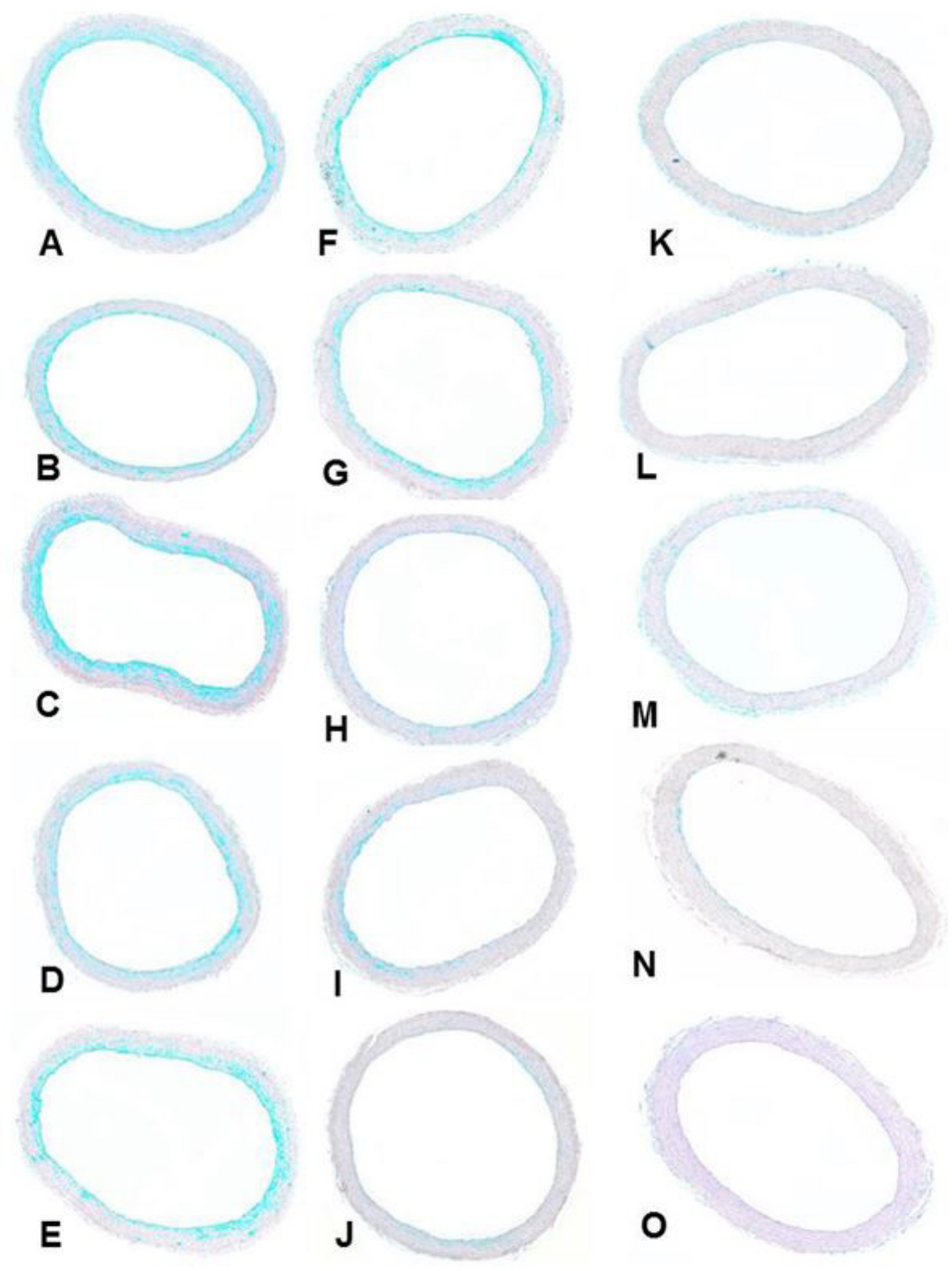

Figura 2: Fotomicrografias de cortes histológicos das artérias que foram classificadas em ordem alfabética, conforme a expressão da quantidade de mucopolissacarídeo presente entre as túnicas, onde letra A representa maior expressão e letra O representa menor expressão. Aumento de 40X. 
Após colocar a identificação das ratas e seus respectivos grupos, notamos que os resultados encontrados nessa análise foram semelhantes aos encontrados nas aferições, onde o G3 representa o maior percentual de depósito de mucopolissacarídeo (Figuras 3 e 4), o GC o menor percentual e o G2 apresenta artérias das ratas com percentual de semelhança dividido entre o G3 e GC (Figura 3 e 4).
Os grupos delineados no trabalho (GC, G2 e G3) foram correlacionados, através do teste de Pearson, com outras variáveis, como a espessura das túnicas arteriais (representada pela diferença entre a área total da artéria e a área da luz do vaso), o diâmetro da luz do vaso e a expressão de mucopolissacarídeos. Foi obtido $r$ de Pearson 0,9830 quando comparadas a medida linear e a expressão de mucopolissacarídeos,
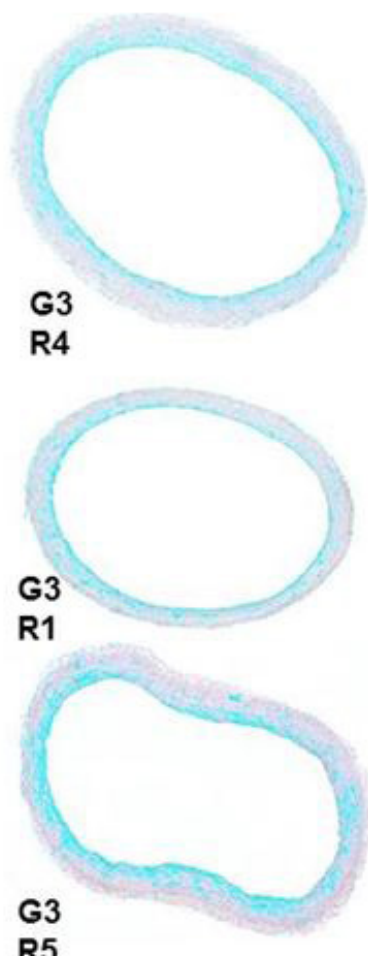

R5

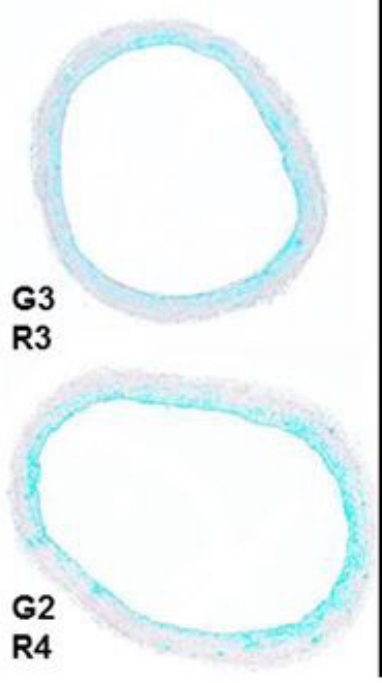

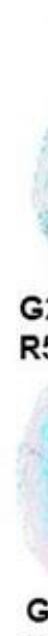

R2

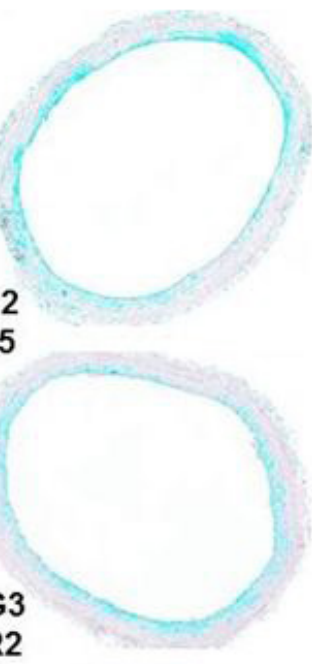

$$
\text { R2 }
$$

G2
R1

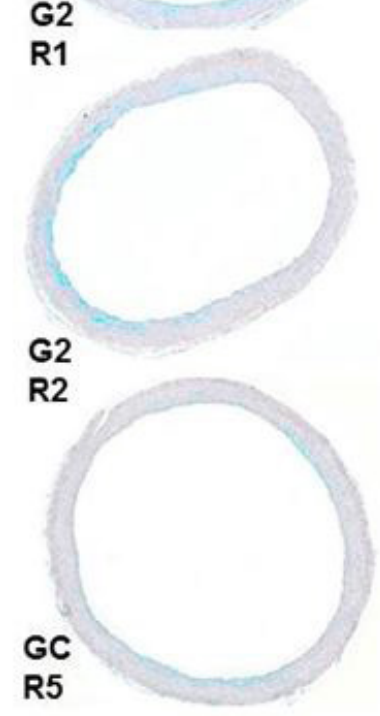

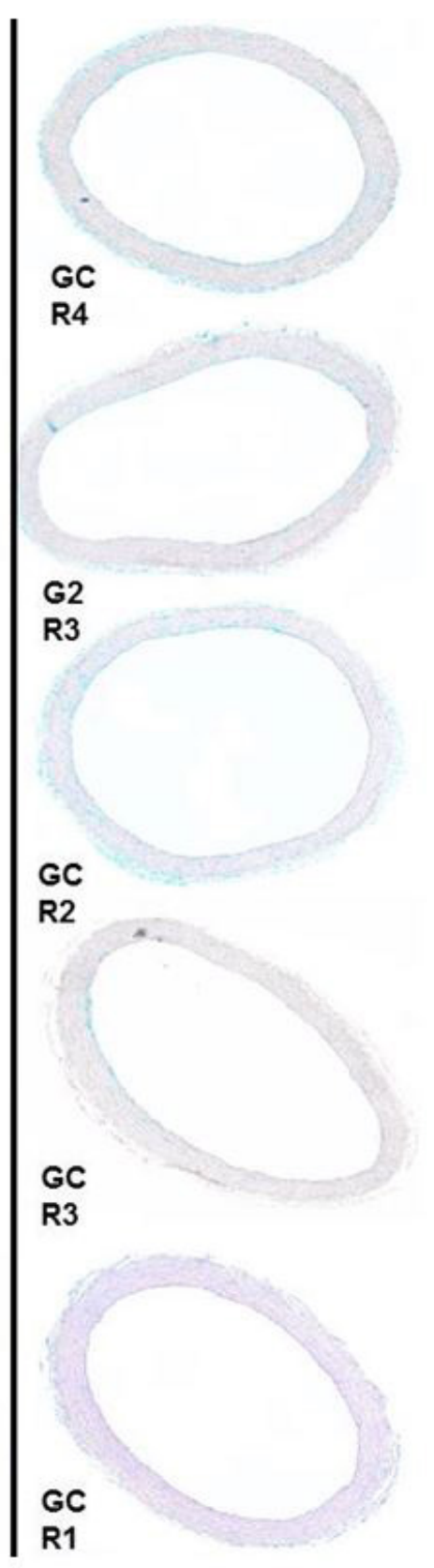

Figura 3: Fotomicrografias de cortes histológicos das artérias que foram organizadas, conforme a expressão da quantidade de mucopolissacarídeo presente entre as túnicas, identificando a qual rata e grupo pertencem as artérias avaliadas, onde a de maior expressão é a artéria da rata (R4) do grupo 3 (G3), e a artéria de menor expressão é a artéria rata (R1) do grupo controle (GC). Aumento de 40X. 


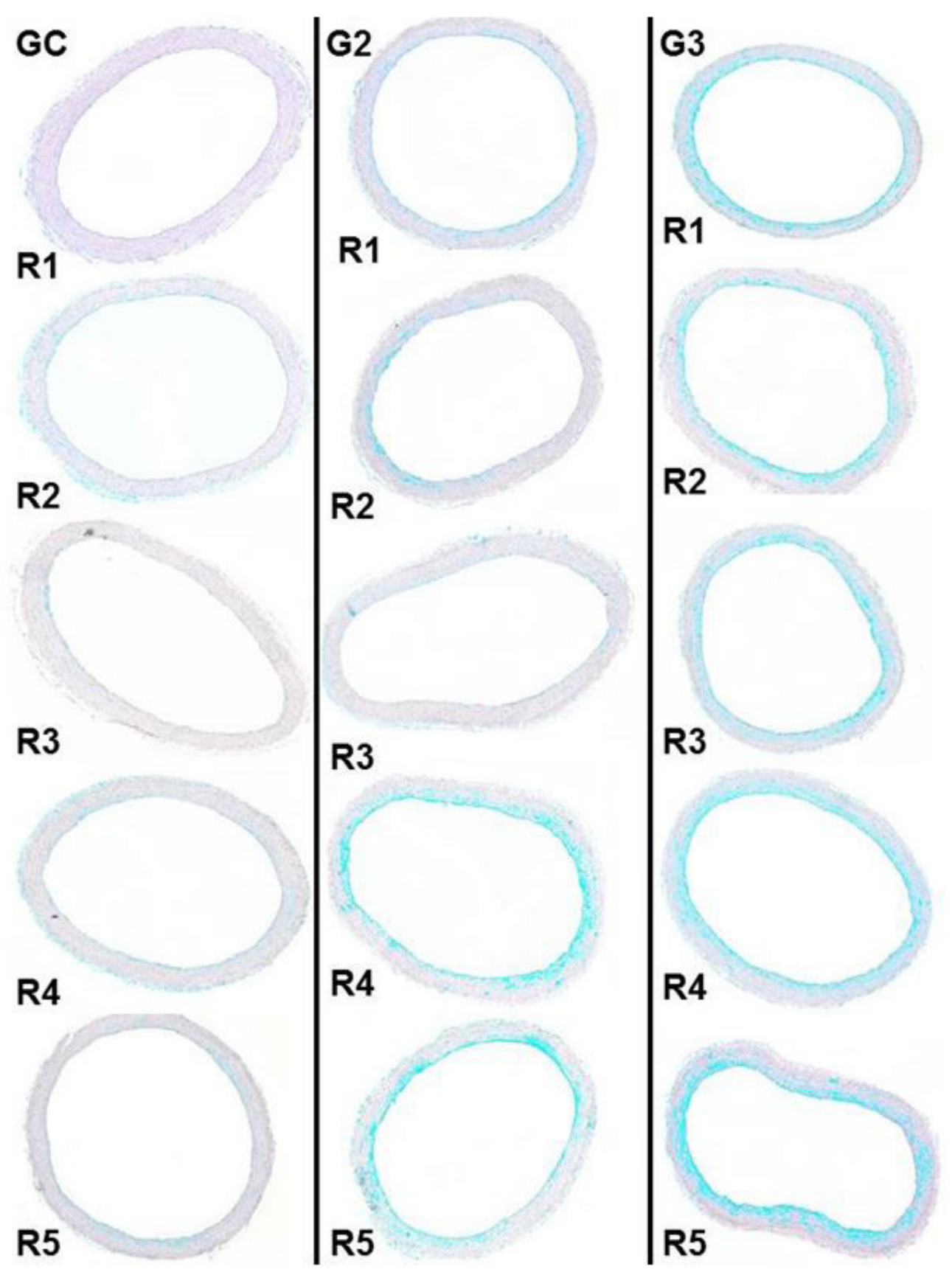

Figura 4: Fotomicrografias de cortes histológicos das artérias que foram organizadas por grupo e animais experimentais, onde a coluna da esquerda apresenta as artérias das 5 ratas do grupo controle (GC), a coluna do meio apresenta as artérias das 5 ratas do grupo 2 (G2) e a coluna da direita apresenta as artérias das 5 ratas do grupo 3 (G3). Aumento de $40 X$.

evidenciando que quanto maior a espessura da artéria, maior a expressão de mucopolissacarídeos. No caso da diferença entre as áreas externa e interna foi obtido $r$ de Pearson 0,9759. Ambas as correlações possuíam $\mathrm{p}<0,0001$. A Figura 5 sintetiza a análise estatística utilizada.
Foi possível realizar uma avaliação simultânea dos resultados encontrados das análises das áreas internas, externas, diâmetro e da expressão de mucopolissacarídeos na artéria aorta. A Figura 6 demonstra os valores das amostras de cada animal experimental dos grupos estudados. 


\begin{tabular}{|c|c|c|c|c|c|c|}
\hline & 1 e 2 & 1 e 3 & 1 e 4 & 2 e 3 & 2 e 4 & 3 e 4 \\
\hline$n$ & 15 & 15 & 15 & 15 & 15 & 15 \\
\hline r Pearson & 0,8502 & 0,8704 & 0,8678 & 0,9830 & 0,9759 & 0,9836 \\
\hline IC $95 \%$ & $0,66-0,95$ & $0,65-0,96$ & $0,64-0,96$ & $0,95-0,99$ & $0,93-0,99$ & $0,95-0,99$ \\
\hline IC $99 \%$ & $0,47-0,96$ & $0,53-0,97$ & $0,52-0,97$ & $0,93-1,0$ & $0,90-0,99$ & $0,93-1,0$ \\
\hline (p) & $<0,0001$ & $<0,0001$ & $<0,0001$ & $<0,0001$ & $<0,0001$ & $<0,0001$ \\
\hline Poder 0,05 & 0,9966 & 0,9986 & 0,9984 & 1,0000 & 1,0000 & 1,0000 \\
\hline Poder 0,01 & 0,9787 & 0,9892 & 0,9881 & 1,0000 & 1,0000 & 1,0000 \\
\hline
\end{tabular}

Figura 5: Os números 1, 2, 3 e 4 indicam as variáveis correlacionadas estatisticamente com uso do teste de Pearson. Onde, (1) Grupos analisados; (2) Diferença entre a área externa e interna; (3) Espessura; (4) Depósito de mucopolissacarídeo.

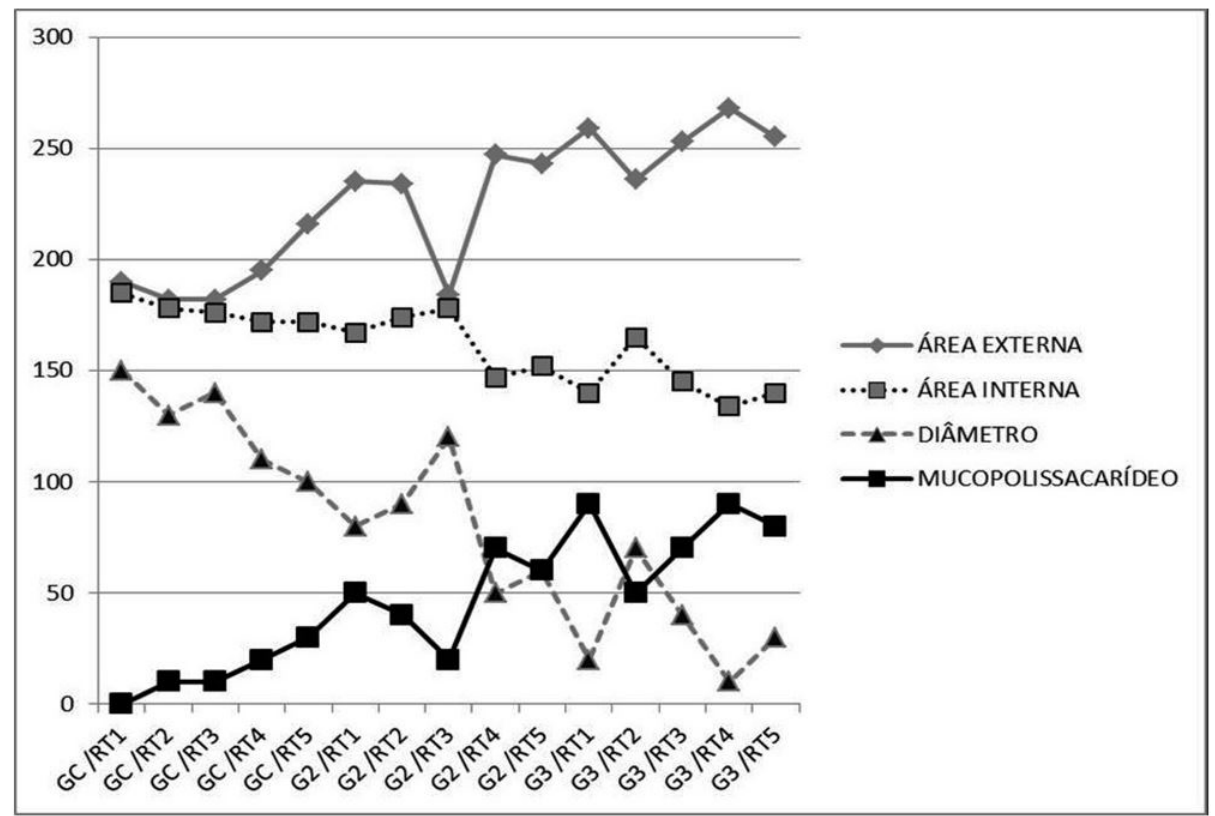

Figura 6: Demonstra resultado da análise individual das amostras de cada animal experimental dos grupos estudados. Onde o resultado apresenta a relação entre o aumento da área externa, a diminuição da área interna, com o aumento do depósito de mucopolissacarídeo e a redução do diâmetro das artérias.

\section{DISCUSSÃO}

Os valores encontrados no presente trabalho demonstram que no grupo tratado com uma dosagem superior de hormônio, G3, há $8 \%$ de amostras com aumento relevante da espessura de suas artérias, ou seja, superior ao grupo que recebeu dosagem menor de hormônio (G2) e ao grupo que não recebeu nenhum tratamento (GC). Os dados também permitem observar que mesmo com dosagem menor de hormônios, o G2 apresenta valores onde 4\% das amostras estão com espessuras de artérias superiores ao GC. Mesmo com um período curto de tratamento, os valores encontrados são corroborados com dados apresentados por Pereira e Angonesi ${ }^{18}$.
A utilização de contraceptivos tem se relacionado intimamente com a descrição de eventos cardiovasculares em pacientes que mantinham uso de anticoncepcionais à base de estrógeno e progesterona. Em relação ao etinilestradiol, por exemplo, alguns estudos demonstraram que ele induz a alterações significativas no sistema de coagulação, culminando com aumento da geração de trombina, ocorrendo também aumento dos fatores de coagulação (fibrinogênio, VII, VIII, IX, X, XII e XIII) e redução dos inibidores naturais da coagulação (proteína $\mathrm{S}$ e antitrombina), produzindo um efeito pró-coagulante leve ${ }^{19,20}$. Esta alteração levaria a um aumento do risco de desenvolvimento de trombose e, 
consecutivamente, à ocorrência de eventos vasculares agudos, como acidentes vasculares isquêmicos agudos (AVI), tromboembolismo pulmonar (TEP) e trombose venosa profunda (TEP). A associação descrita é muito similar aos resultados encontrados.

Com um fator de correlação significativo demonstrou-se que a utilização de hormônios contraceptivos induz ao espessamento arterial e leva a uma redução da luz vascular. Este achado soma-se às possíveis modificações de hipercoagulabilidade associadas ao uso de hormônios estrogênicos, acentuando o risco para o desenvolvimento de problemas vasculares. Alterações nas túnicas e na luz das artérias são alterações morfológicas que também podem estar relacionadas às alterações morfológicas encontradas nesse estudo. Percebe-se uma íntima relação entre as doses utilizadas nos anticoncepcionais administrados e o aumento da espessura das túnicas arteriais, em função do depósito de mucopolissacarídeos. Em alguns estudos, a aterosclerose foi caracterizada pelo espessamento da túnica, devido ao aumento de seu volume no interior das camadas ${ }^{21}$.

Conforme relatos de trabalhos publicados, as artérias têm a parede espessada à custa de hipertrofia da túnica média e fibrose da íntima. Outros fatores que acabam por obstruir a luz do vaso são os relacionados à proliferação dos chamados miofibroblastos, que são células pouco diferenciadas derivadas de células musculares migradas a partir da túnica média, e da deposição de matriz extracelular (colágeno, elástica, proteoglicanos) sintetizada pelas mesmas células ${ }^{22}$. Esses relatos confirmam os achados do presente trabalho, o qual obteve espessamento significante das túnicas, relacionado com o depósito de mucopolissacarídeos presente nas mesmas.
Em nossos achados esta relação se deve, provavelmente, pelo efeito sistêmico dos hormônios estudados, que induzem, em doses aumentadas, uma maior expressão de alguns receptores celulares associados ao metabolismo dos mucopolissacarídeos, o que, com o uso continuado, levaria ao depósito destas substâncias nas artérias e, consecutivamente, ao espessamento vascular, no entanto, esta relação não é bem descrita na literatura. Apesar de muitos artigos evidenciarem a possibilidade de contraceptivos hormonais potencializarem o risco para eventos cardiovasculares ${ }^{23}$, pouco foi estudado sobre depósitos de mucopolissacarídeos em artérias, sob efeito de doses hormonais anticoncepcionais.

As análises do presente trabalho demonstram que os anticoncepcionais de ultrabaixa e baixa dosagem influenciaram nas propriedades morfofuncionais das artérias.

A coloração histoquímica de Verhoeff foi eficiente na identificação das fibras elásticas, onde não evidenciamos alterações significantes entre os grupos estudados. A técnica de aferição em HE e a coloração específica de histoquímica com Alcian Blue permitiram evidenciar alterações significantes entre os grupos estudados, demonstrando relações entre o espessamento das artérias com a grande quantidade de mucopolissacarídeos depositados entre as túnicas das mesmas.

\section{Agradecimentos}

Os autores apreciam a generosa disponibilidade do A.C. Camargo Cancer Center, que possibilitou a realização do procedimento de coloração específica em histoquímica no seu serviço de patologia anatômica.

\section{Conflitos de Interesse}

Os autores declaram não ter conflitos de interesse.

\section{REFERÊNCIAS}

1. Bahamondes L, Pinho F, Melo RN, Oliveira E, Bahamondes VA. Fatores associados à descontinuação do uso de anticoncepcionais orais combinados. Rev Bras Ginecol Obstet. 2011;33(4):3003-9.

2. Baracat EC, Barbosa IC, Campos AA, Hyppolito SB, Melo NR, Mussielo R, et al. Avaliação da tolerabilidade e do controle de ciclo de dois contraceptivos orais de baixa dose: estudo comparativo aberto. Rev Bras Ginecol Obstet. 1998;20(5):273-80.
3. Wilde MI, Balfour JA. Gestodene: a review of its pharmacology, efficacy and tolerability in combined contraceptive preparations. Drugs. 1995;50(2):364-95. http://dx.doi. org/10.2165/00003495-19955002000010. PMid:8521763.

4. World Health Organization (WHO). Venous thromboembolic disease and combined oral contraceptives: results of international multicentre case-control study. Lancet. 1995;346(8990):157582. http://dx.doi.org/10.1016/S01406736(95)91926-0. PMid:7500748.
5. Carneiro AFGC. Influência do ciclo cardíaco no fluxo sanguíneo na vizinhança da bifurcação ilíaca [tese]. Portugal: Universidade do Minho; 2009.

6. Brasileiro G Fo. Bogliolo: patologia. 8. ed. Rio de Janeiro: Guanabara Koogan; 2011. 1524 p.

7. Stary HC, Chandler AB, Dinsmore RE, Fuster V, Glagov S, Insull W $J R$, et al. A definition of advanced types of atherosclerotic lesions and a histological classification of atherosclerosis. Circulation. 
1995;92(5):1355-74. http://dx.doi. org/10.1161/01.CIR.92.5.1355.

PMid:7648691.

8. Stary $\mathrm{HC}$, Blankenhorn $\mathrm{DH}$, Chandler AB, Glagov S, Insull W JR, Richardson $\mathrm{M}$, et al. A definition of the intima of human arteries and of its atherosclerosis-prone regions. Circulation. 1992;85(1):391-405. http:// dx.doi.org/10.1161/01.CIR.85.1.391. PMid:1728483.

9. Robbins SL. Patologia estrutural e funcional. 6. ed. Rio de Janeiro: Guanabara Koogan; 2000. 1251 p.

10. Willis GCS. Intimal ground substance in atherosclerosis. Can Med Assoc J. 1953;69(1):17-22. PMid:13059725.

11. Pedrosa VF, Romano LA, Santos FL, Guimarães JM, Silva ADR, Mendes ES. Hiperplasia em túnicas de artérias coronárias de beijupirás criados em sistema offshore. Arq Bras Med Vet Zootec. 2015;67(3):747-54. http:// dx.doi.org/10.1590/1678-4162-7497.

12. Kumar $\mathrm{V}$, Abbas AK, Fausto $\mathrm{N}$, Aster JC. Robbins e Cotran: patologia: bases patológicas das doenças. 8. ed. Rio de Janeiro: Elsevier; 2010. 1458 p.

13. Junqueira LCU, Carneiro J. Histologia básica: texto e atlas. 12. ed. Rio de Janeiro: Guanabara Koogan; 2008. $524 \mathrm{p}$.
14. Junqueira LCU, Bignolas G, Brentani RR. Picrosirius staining plus polarization microscopy, a specific method for collagen detection in tissue sections. Histochem J. 1979;11(4):447-55. http:// dx.doi.org/10.1007/BF01002772. PMid:91593.

15. Tolosa EM, Rodrigues CJ, Behmer AO, Freitas AG No. Manual de técnicas para Histologia normal e patológica. 2. ed. São Paulo: Manole; 2003.

16. Caputo LFG, Gitirana LB, Manso PPA. Técnicas histológicas. In: Molinaro EM, Caputo LFG, Amendoeira MRR, editores. Conceitos e métodos para a formação de profissionais em laboratório. Rio de Janeiro: Fiocruz; 2010. p. 90-188. (vol. 2).

17. Brasil. Ministério da Saúde do Brasil. Secretaria de Atenção a Saúde. Protocolos clínicos e diretrizes terapêuticas. 2. ed. Brasilia: 2010. (Serie A. Normas e Manuais Técnicos) [citado $2018 \mathrm{Fev}$ 02]. Disponível em: http://bvsms. saude.gov.br/component/content/ article?layout=edit\&id=2281.

18. Pereira PVS, Angonesi D. Efeitos do uso prolongado de contraceptivos orais. Infarma-Ciên. 2009;21(7-8):218.
19. Mammen EF. Oral contraceptive pills and hormonal replacement therapy and thromboembolic disease. Hematol Oncol Clin North Am. 2000;14(5):1045-59, vii-viii. http://dx.doi.org/10.1016/S08898588(05)70170-2. PMid:11005033.

20. Rosendaal FR, Van Hylckama Vlieg A, Tanis BC, Helmerhorst FM. Estrogens, progestogens and thrombosis. $J$ Thromb Haemost. 2003;1(7):137180. http://dx.doi.org/10.1046/j.15387836.2003.00264.x. PMid:12871270.

21. Moreno H JR, Metze K, Bento AC, Antunes E, Zatz R, Nucci G. Chronic nitric oxide inhibition as a model of hypertensive heart muscle disease. Basic Res Cardiol. 1996;91(3):248-55. http://dx.doi.org/10.1007/BF00788911. PMid:8831944.

22. Rabinovitch M. Pathobiology of pulmonary hypertension: extracellular matrix. Clin Chest Med. 2001;22(3):433-49, viii. http://dx.doi. org/10.1016/S0272-5231(05)70282-3. PMid:11590839.

23. Augsburger HR, Kurzi M. Histomorphologic and morphometric evaluation of the uterine horns in nulliparous and multiparous beagles. Am J Vet Res. 2004;65(5):5528. http://dx.doi.org/10.2460/ ajvr.2004.65.552. PMid:15141872.

Recebido: 2 fev, 2018 Aceito: 11 jun, 2018 